\title{
The Research on Adaptive Control Modeling of a Liquid Fertilizer Spreader
}

\author{
Zidong Yang \\ School of Agriculture Engineering and Light Industry, Shandong University of Technology, Shandong, China \\ E-mail:Yzd@sdut.edu.cn \\ Received September 28, 2009; revised October 22, 2009; accepted October 28, 2009
}

\begin{abstract}
This paper describes a general modeling and control approach for steering wheel variable rate liquid fertilizer applicator. An adaptive numerical modeling approach for describing the system input-output dynamics is proposed, and an optimal control that accounts for the control hardware limits is developed. Field tests have demonstrated the effectiveness of the theoretical development.
\end{abstract}

Keywords: Adaptive Control, Variable Rate Fertilizer, Precision Agriculture, Optimal Control, Manure Spreader

\section{Introduction}

Based on a set of high-new technologies, such as the modern information technology, the organism technology and the engineering technology, etc., the precision agriculture has become the important way of modern agricultural production. Compared with foreign developed countries, the intensive level is quite low in China. However, according to the characteristics of the agricultural development in China, the technological system of water-saving and variable rate fertilizer should be developed in the near future. The precision equipped agriculture can be implemented firstly in the region where the equipped agriculture has been developed fast. For example, the big farms, which have large scales and high mechanization level, may carry on the practice of the precision agriculture.

Fertilizer-saving precision agriculture can not only decrease costs, but also increase yields. Furthermore, accurately applying chemicals and fertilizers only where needed can reduce the potential for ground and surface water pollution. Manure produced by livestock contains valuable nutrients for crops. Additional fertilizers are often applied to increase the crop production. Excessive applied manure and fertilizer contributes to ground and surface water pollution and also increases the cost of crop production. So there is a need to develop an automated spreader in order to achieve consistent and precise application of crop nutrients.

Straub et al. (1998) described a computer controlled manure spreader developed by John Deere Corporation in collaboration with the University of WisconsinMadison, and carried out field tests indicating that the control system worked well with lighter and dryer manure. One of the problems they faced is how to measure the manure discharge. In their control system, the weight of the spreader is measured over time and a finite difference method is used to compute the discharge rate. High-performance controllers, including a supervisory control and a control with a Kalman filter and a Smith predictor for time delay, have been developed by [1]. Magnetic inductive flow meters are used to measure the manure's flow rate. Landry et al. [2,3] recently studied physical and rheological properties of manure and investigated the effectiveness of conveying systems for manure spreaders.

The present study is on a system on-line identification algorithm. A numerical regression model is designed to describe the input-output dynamics of the spreader. The parameters of the numerical model are updated in real time to account for the time varying and nonlinear properties of the spreader dynamics.

The remainder of the paper is organized as follows: in the section below, we describe the objectives of the research and a description of the hardware and software system. Then we present a discussion of numerical modelling of the input-output dynamics of the spreader and an experimental validation of the model. The adaptive optimal control for regulating the discharge rate of the spreader is subsequently developed.

\section{Research Objectives and System Description}

Because China has a large number of small and medium sized tractors, in order to increase output, the mulch 
sowing and straw returning has been widely popularized. But this has caused some difficulties for variable-rate fertilization and deep fertilization of the liquid fertilizer. The general variables spraying method can't adapt to this situation. With regards to this, we have designed a steering wheel variable rate manure spreader and the adaptive control system, which is more suitable for medium-small size tractors, variable-rate fertilization and deep fertilization to the liquid fertilizer. A picture of the machine is shown in Figure 1. The auger speed and the gate opening size can be controlled. The objective of the control algorithm is to regulate these two quantities for a pre-determined spreading application density per unit area. As an example, the control task set for the present study is to attain a specified constant discharge mass per unit area from the spreader taking into account varying speed of the tractor and the material variability of the semi-solid animal wastes. This manure spreader used variable rate technologies (VRT) describes machines that can automatically change their application rates in response to their position.

The core of the VRT system is the flow rate controller. Essentially, the flow control system receives the set point flow rate from the application system (likely a GPS/GIS system) on-board the tractor and then manipulates a number of actuators in an attempt to adjust the actual flow-rate to match the set-point.

To provide a specific illustration, consider the diagram of a relatively simple liquid sprayer VRA system as depicted in Figure 2. The following discussion is provided as one scenario for each component, but there may be alternative sensors and methods of control. A radar based ground speed sensor would be used to provide true ground speed to the computer/controller since application rate is a function of speed. This system depicts the use of a direct injection sprayer, which is the direction in

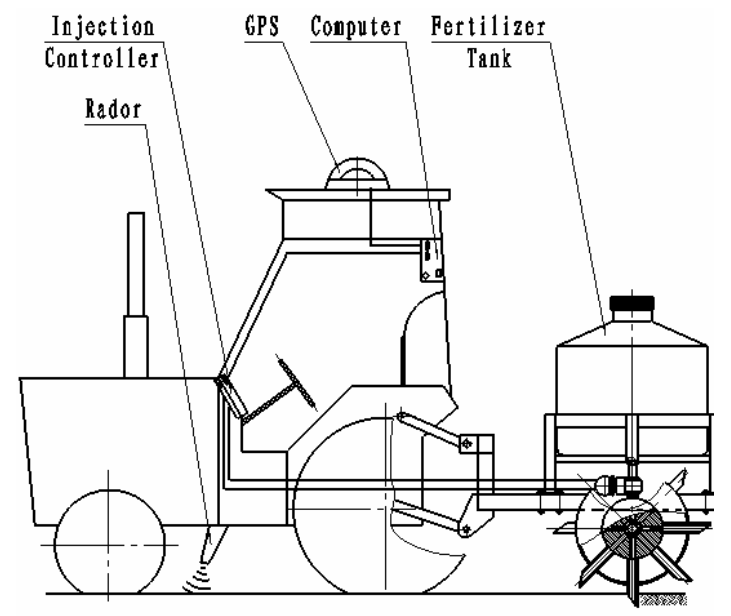

Figure 1. Schematic representation of the components of a VRT manure spreader. which sprayer technology is proceeding. With this type of sprayer, the operator does not mix the chemical(s) in the main tank, rather, the chemical(s) remains in a container, where it may be pumped as needed into an injector where the chemical(s) is automatically mixed with water on-the-fly. There are many advantages to this system as compared with tank-mixing, such as safety, managing mixed chemicals, and automation. The injector pump may be designed to provide precise control of the injection rate of the chemical concentrate to the injector. The water tank may have a level sensor which will allow the computer/controller to determine the amount of water remaining in the tank in gallons. The total flow rate of the fluid going to the boom(s) will be controlled by the flow control valve, which in turn is controlled by the computer/controller. The actual total fluid flow rate will be monitored by the fluid flow rate sensor, and this information will be used by the computer/controller for fine adjustments in the flow control valve. The fluid flow rate and the vehicle position will be continuously recorded in the computer as the vehicle sprays to provide a historical record for the GIS about where and how much chemical was dispensed. The boom valve will be used to turn the boom on or off to provide fast accurate control of the application area.

The controllers are very similar to those used on many sprayers, spreaders and other agricultural machines. On conventional machines, the operator controls the application rate by selecting the desired rate from the console panel in the cab. It is assumed that the spreading width of the material is a constant. Note that the auger speed is adjusted by varying the swash plate angle of the

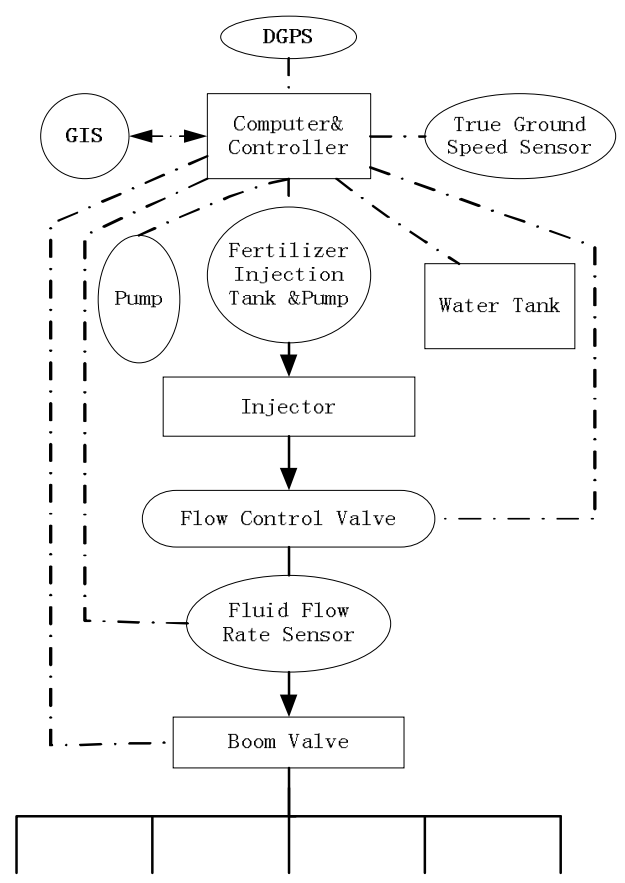

Figure 2. The sketch map of liquid fertilizer sprayer. 
hydraulic pump. In order to develop control algorithms to achieve the above objective, we must first develop a dynamic model for the spreader. Specifically, we need a relationship between the input, i.e. the auger speed and the gate opening size, and the output, i.e. the material discharge rate. Recall that the material is a highly inhomogeneous mix of liquids and solids with unknown percentage of each phase. The weight and viscosity of the material affect the dynamics of the hydraulic system that drives the auger. As the spreading proceeds, the amount of material remaining in the tank changed. All these factors attribute to a nonlinear and time-varying dynamics of the spreader. As discussed in Section 1, analytical modeling of such a system is a difficult task. In this study, we propose to develop an on-line numerical model of the input-output relationship, known as the system transfer function. The controller of on-line model has an Atmel processor $89 \mathrm{~S} 51$ with $33 \mathrm{MHz}$ frame rate. It communicates with a laptop computer via RS232 at 9600 baud. This controller can interface with and control a wide range of equipment including variable rate applicators for precision agriculture. The on-line system model fits the experimental data to a pre-determined numerical model with undetermined coefficients. A very common numerical model can describe a large.

\section{On-Line System Modeling}

Class of dynamic systems is the autoregressive model with exogenous inputs (ARX) (Billings, 1986; Diaz and Desrochers, 1988; Ljung, 1987). It is given in a general form as

$$
\begin{aligned}
& y_{n}+a_{1} y_{(n-1)}+\ldots+a_{n_{a}} y_{\left(n-n_{a}\right)} \\
& =b_{1} u_{\left(n-n_{k}\right)}+\ldots+b_{n_{b}} u_{\left(n-n_{k}-n_{b}+1\right)}
\end{aligned}
$$

The current output $y_{n}$ is assumed to be a function of a finite history of output values $y_{(n-1)}$ to $y_{\left(n-n_{a}\right)}$ and the delayed input $u_{\left(n-n_{k}\right)}$ to $u_{\left(n-n_{k}-n_{b}+1\right)}$. The coefficients $a_{i}\left(i=1, \ldots, n_{a}\right)$ and $b_{j}\left(j=1, \ldots, n_{b}\right)$ are undetermined. The on-line modeling algorithm determines the coefficients and approximates the numerical model to the measured system dynamics in some optimal manner.

Strictly speaking, the ARX model is valid for linear dynamic systems. The present spreader system is time varying and nonlinear. A properly identified ARX model will accurately represent the dynamics of the system over a short time interval and will not be valid for the entire history of the spreading task from a full tank to empty. Therefore the ARX model must be updated frequently during spreading. Efficient real-time adaptive algorithms will be needed for this task.

\subsection{Adaptive Algorithm}

In signal processing, the ARX model is also known as an infinite impulse response (IIR) filter (Haykin, 1991).A popular steepest gradient descent method known as the least mean square (LMS) algorithm (Widrow and Stearns, 1985) can be used to adjust the coefficients of the ARX model and minimize the error between the prediction of the numerical model and the real measurement. The estimation error is $e_{k}=d_{k}-y_{k}$, where $d_{k}$ is the measured output and $y_{k}$ is the predicted output. A performance index can be defined as $J(k)=e_{k}^{2}$. We write the ARX model in a vector notation as

$$
y_{k}=\mathfrak{I}_{w}^{T} \boldsymbol{I}_{k}
$$

where $\mathbf{I}_{k}^{T}$ is a vector consisting of the undetermined coefficients at the kth time step and $\dot{u}_{k}$ is a vector consisting of both the past history of $y_{k}$ and the control inputs. The LMS algorithm for updating the undetermined coefficients in order to minimize $J(k)$ is given by

$$
\stackrel{\prime}{w}_{(k+1)}=\stackrel{\prime}{w}_{k}+\beta e_{k} \stackrel{\prime}{u}_{k}
$$

where $\beta$ is an adaptation gain parameter.

\subsection{Experimental Validation of the LMS Algorithm}

We have selected a simple ARX model for the spreader given by

$$
y_{k}=a_{1} y_{(k-1)}+a_{2} u_{(k-1)}^{s}+a_{3} u_{(k-1)}^{g}
$$

where $u_{(k-1)}^{s}$ denotes the swash plate angle that regulates the auger speed and $u_{(k-1)}^{g}$ is the rear gate opening. We have carried out experiments to compare this simple model with more complicated ones, and found that this model describes the system with a good balance of accuracy and efficiency, and is sufficient for our work. A digital second order IIR low pass filter of bandwidth $2 \mathrm{~Hz}$ programmed in the $\mathrm{C}$ language is used to block noise in the weight signal. The weight signal is sampled at a rate of $100 \mathrm{~Hz}$ in real time. During spreading, the gate positions are fixed for a period of time during which the swash plate is swept from being completely closed to being fully open to adjust the auger speed. The purpose of doing so is to create a set of data from one test run that excites as much of the system dynamics as possible. The model prediction is seen to be quite accurate. The parameter $a_{1}$ is nearly equal to one. This is physically reasonable since in the absence of the control, i.e. when the auger speed is zero and the gate is closed, the material remaining in the tank is unchanged. The parameters $a_{2}$ and $a_{3}$ are negative. It is again physically reasonable that $a_{2}$ and $a_{3}$ are negative. When the control inputs are greater than zero, the material remaining in the tank $y_{k}$ decreases. The ranges of these coefficients are as follows: 
$\max \left(a_{1}\right)=1.0047, \min \left(a_{1}\right)=0.9881$, average $a_{1}=1.0011 ;$ $\max \left(a_{2}\right)=-0.0083, \min \left(a_{2}\right)=-0.0111$, average $a_{2}=-0.0094$; $\max \left(a_{3}\right)=-0.0045, \min \left(a_{3}\right)=-0.0080$, average $a_{3}=-0.0063$.

\section{Control Algorithm}

The control algorithm design depends on the system model. This section focuses on a discussion of the control algorithm design. The integration of the control loop and the parameter updating loop is natural and is coded in the software.

\subsection{Range Limited Optimal Control}

Lewis and Syrmos [4] have shown that after going through the steps of optimal control solutions and taking $i=k$ and $N=k+1$, we obtain the unconstrained optimal control solution as

$$
u_{k}^{*}=-\left(R+c_{k} c_{k}^{T} s N\right)^{-1} c_{k} s N\left(a_{1} e_{k}+G_{k}\right)
$$

We shall continue the study with the one step optimal control. Recall that the range of $u_{k}^{s}$ and $u_{k}^{g}$ is finite. The unconstrained optimal solution (5) is valid when the bound is not exceeded. To account for the bounds on the controls, we need to use the Pontryagin's minimum principle. This leads to the following in equality for determining the control $u_{k}^{*}$ :

$$
\frac{1}{2} u_{k}^{* T} R u_{k}^{*}+\lambda_{k+1}^{*} c_{k}^{T} u_{k}^{*} \leq \frac{1}{2} u_{k}^{T} R u_{k}+\lambda_{k+1} c_{k}^{T} u_{k}
$$

The inequality holds for all admissible values of $u_{k}$. The optimal control can be found from the inequality by considering an auxiliary problem of minimization of the following quadratic form:

$$
w=\frac{1}{2}\left(u_{k}+R^{-1} c_{k} \lambda_{k+1}\right)^{T}\left(u_{k}+R^{-1} c_{k} \lambda_{k+1}\right)
$$

It can be shown that the $u_{k}$ that minimizes $w$ also minimizes the left hand size of the inequality (5). Let the lower and upper bounds of the control be denoted by $u_{k_{\min }}^{s, g}$ and $u_{k_{\max }}^{s, g}$. Let $u_{k}^{s, g}$ denote the swash plate and gate opening control elements of the vector in Equation 5, i.e.:

$$
u_{k}^{s, g}=\left[-\left(R+c_{k} c_{k}^{T} s N\right)^{-1} c_{k} s N\left(a_{1} e_{k}+G_{k}\right)\right]^{s . g}
$$

After several algebraic steps, we obtain the range constrained optimal control as

$$
u_{k}^{*_{s, g}}=\left\{\begin{array}{c}
u_{k_{\min }}^{s, g}, U_{k}^{s, g} \leq u_{k_{\min }}^{s, g} \\
U_{k}^{s, g}, u_{k}^{s, g}<U_{k}^{s, g}<u_{k_{\max }}^{s, g} \\
u_{k_{\max }^{s, g}}^{s, g}, U_{k}^{s, g} \geq u_{k_{\max }^{s}}^{s, g}
\end{array}\right.
$$

The middle branch of the solution is the same as that in Equation 5. In other words, when the system operates within the physical limits of the controls, the solution given by Equation 5 is optimal. Note that when the num- ber of inputs is greater than the number of outputs, the matrix $\mathrm{R}$ cannot be zero, and has to be positive definite.

\subsection{Rate Limited Optimal Control}

The range limited optimal control problem implies that the controls can be instantly switched from one level to another. This is of course not realistic since a physical device always takes a finite time to change and has inherent delays. When the controller requires the system to change faster than the physical rate limit, rate saturation occurs. To account for the rate limits, we once again invoke the Pontryagin's minimum principle and consider the increment $\Delta u_{k-1}$ such that $u_{k}=u_{k-1}+\Delta u_{k-1}$ as the control variable. Applying the Pontryagin's minimum principle in terms of the control increment, we have another inequality

$$
\begin{aligned}
& \frac{1}{2}\left(u_{k-1}^{*}+\Delta u_{k-1}^{*}\right)^{T} R\left(u_{k-1}^{*}+\Delta u_{k-1}^{*}\right)+\lambda_{k+1}^{*} c_{k}^{T}\left(u_{k-1}^{*}+\Delta u_{k-1}^{*}\right) \\
& \leq \frac{1}{2}\left(u_{k-1}+\Delta u_{k-1}\right)^{T} R\left(u_{k-1}+\Delta u_{k-1}\right)+\lambda_{k+1} c_{k}^{T}\left(u_{k-1}+\Delta u_{k-1}\right)
\end{aligned}
$$

The optimal control increment can be found from the inequality by considering an auxiliary problem of minimization of the following quadratic form:

$$
w=\frac{1}{2}\left(u_{k-1}+\Delta u_{k-1} R^{-1} c_{k} \lambda_{k+1}\right)^{T}\left(u_{k-1}+\Delta u_{k-1}+R^{-1} c_{k} \lambda_{k+1}\right)
$$

Define an increment by using Equation 8 as

$$
\Delta U_{k}^{s, g}=U_{k}^{s, g}-U_{k-1}^{s . g}
$$

By minimizing $w$ with respect to $\Delta u_{k-1}$, we obtain the optimal control increment as

$$
\Delta u_{k-1}^{* s, g}=\left\{\begin{array}{cl}
\Delta U_{k}^{s, g} & \left|\Delta U_{i, k}\right| \leq \Delta u_{k \max }^{s, g} \\
\operatorname{sgn}\left(\Delta U_{k}^{s, g}\right) \Delta u_{k \max }^{s, g} & \left|\Delta U_{i, k}\right|>\Delta u_{k \max }^{s, g}
\end{array}\right.
$$

where $\Delta u_{k_{\max }}^{s, g}$ denotes the physically allowable maximum rate of change of the swash plate and rear gate controls over one sample interval. The top branch of the solution matches the range limited optimal control and the lower branch is the rate saturated control. By combining Equations 5 and 13, we obtain the optimal control under both range and rate saturation limits. More discussions of such optimal control problems can be found in Kobs and Sun (1997).

\section{Discussions and Conclusions}

In order to attain the goal of saving fertilizer, cutting down production cost and protecting environment, the liquid fertilizer applicator was designed and tested in field trial based on its advantages of non-dust, non-smog and reducing environment pollution during the process of production, usage and transportation. The optimized working 
parameters by means of field trial were as follows: fertilization depth of $60-100 \mathrm{~mm}$, operation velocity of $1.3 \mathrm{~m} / \mathrm{s}$ and pump working pressure of $0.36 \mathrm{MPa}$. Let $D_{A}$ denotes the required mass per unit area $\left(\mathrm{kg} / \mathrm{m}^{2}\right)$. The spreading width is $1.5 \mathrm{~m}$. A relationship between the discharge rate $D_{t}$ per unit time $(\mathrm{kg} / \mathrm{s})$, the speed of the tractor $v(\mathrm{~m} / \mathrm{s})$ and $D_{A}$ can be found as $D_{t}=4.167 v D_{A}(\mathrm{~kg} / \mathrm{s})$. Since the discharge rate is constant when the tractor speed is constant, the material remaining in the tank is a linearly decreasing function of time. The actual measurement is in good agreement with the reference input. Note that there is significant noise in the measurement due to vehicle dynamics and electronic disturbances. The low pass digital filter designed for the weight sensor is quite effective in reducing measurement noise.

We have presented a general modeling and control approach for precision agricultural applications by using a SYF-2 manure spreader as an example. The numerical input-output modeling approach can handle a wide range of variations in manure materials and the complicated nonlinear dynamics of the machine. The adaptive selftuning optimal control algorithm can cope with various hardware limits. The theoretical development has been validated by extensive experimental results. The present approach provides a promising methodology for automat- ing machines for precision agricultural applications.

\section{Acknowledgments}

This paper supported by National Key Technology R\&D Program during the 11th Five-Year Plan Period of China (2006BAD11A17) and the visiting scholar project of excellent young teachers of higher learning in Shandong Province.

\section{References}

[1] A. Munack, E. Buning, H. Speckmann, "A high-performance control system for spreading liquid manure," Control Engineering Practice, Vol. 9, pp. 387-391, 2001.

[2] H. Landry, C. Lague, and M. Roberge, "Physical and rheological properties of manure products," Applied Engineering in Agriculture, Vol. 20, No. 3, pp. 277-288, 2004.

[3] H. Landry, E. Piron, J. M. Agnew, C. Lague, M. Roberge, "Performances of conveying systems for manure spreaders and effects of Hopper geometry on output flow," Applied Engineering in Agriculture, Vol. 21, No. 2, pp. 159-166, 2005.

[4] F. L. Lewis, V. L. Syrmos, "Optimal control," John Wiley and Sons, Inc., New York, 1995. 